\title{
Reporting epidemiology of antibiotic resistance
}

\author{
Simone Ambretti, ${ }^{1}$ Carlo Gagliotti, ${ }^{2}$ Francesco Luzzaro, ${ }^{3}$ Paolo Malacarne, ${ }^{4}$ Angelo Pan, ${ }^{2,5}$ \\ Barbara Pieretti, ${ }^{6}$ Carlo Tascini, ${ }^{7}$ Mario Sarti, ${ }^{8}$ Comitato CoSIAS-AMCLI \\ ${ }^{1}$ Microbiology Unit, S.Orsola-Malpighi University Hospital, Bologna; ${ }^{2}$ Infectious Risk Unit, \\ Regional Agency for Health and Social Care of Emilia-Romagna, Bologna; ${ }^{3}$ Microbiology and \\ Virology Unit, Manzoni Hospital, Lecco; ${ }^{4}$ Anaesthesia and Resuscitation Unit, Emergence \\ Department, Pisa University Hospital; ${ }^{5}$ Infectious and Tropical Disease Division, Cremona \\ Hospital, Cremona; ${ }^{6}$ Clinical Pathology Laboratory, S. Croce Hospital, Marche Nord Hospitals, \\ Fano; ${ }^{7}$ Infectious Disease Unit, Pisa University Hospital, Pisa; ${ }^{8}$ Provincial Clinical Microbiology \\ Unit, Modena NOCSAE, Baggiovara, Modena Healthcare Unit, Italy
}

\section{Summary}

The aim of antimicrobial resistance surveillance is to monitor temporal trends and provide clinicians with data to define empirical treatment protocols. The surveillance methods adopted in different settings can be significantly different and, therefore, no reference can be made to a single set of standards. This paper outlines the main features of analysis and reporting of antimicrobial resistance data according to the guidelines issued by the US Clinical and Laboratory Standards Institute, and the surveillance systems adopted in Europe. In this article the strengths and weaknesses of the various types of analyses will be discussed highlighting the critical aspects to be taken into account in surveillance data reporting.

\section{Introduction}

The periodic evaluation of microorganisms isolated from biological cultures and their antimicrobial susceptibility profiles is fundamental

Correspondence: Simone Ambretti, Microbiology Unit, S.Orsola-Malpighi University Hospital, V. Massarenti 9, 40138 Bologna, Italy.

Tel.: +39.051.2144515 - Fax: +39.051 .2143672 .

E-mail: simone.ambretti@aosp.bo.it

Key words: Antimicrobial resistance; antibiogram; surveillance; temporal trends; reports.

Contributions: the authors contributed equally.

Conflict of interest: the authors declare no potential conflict of interest.

Received for publication: 5 June 2015.

Accepted for publication: 5 June 2015 .

(C) Copyright S. Ambretti et al., 2015

Licensee PAGEPress, Italy

Microbiologia Medica 2015; 30:5308

doi: $10.4081 / \mathrm{mm} .2015 .5308$

This article is distributed under the terms of the Creative Commons Attribution Noncommercial License (by-nc 3.0) which permits any noncommercial use, distribution, and reproduction in any medium, provided the original author(s) and source are credited. to monitoring resistance patterns, thus supporting both clinicians in empirical antibiotic therapy decision-making and all healthcare providers involved in infection and antibiotic resistance monitoring when selecting actions to implement and assessing their efficacy.

Epidemiological surveillance activities by microbiology laboratories have gained growing importance given the spread of antibiotic resistance and currently play a pivotal role in antimicrobial stewardship programs. In its guide for the prevention of hospital-acquired infections, the World Health Organization (WHO) indicates among the formal tasks of microbiologists that they should be responsible for providing periodic reports of antimicrobial susceptibility patterns (9).

Resistance patterns of the main pathogens are generally monitored and reviewed on a yearly basis to calculate their susceptibility rates through the so-called cumulative antibiogram. The evaluation of these data can be based on different algorithms, thus generating inevitably incomparable data, as shown by the outcome of a survey conducted back in 2000 by the Clinical and Laboratory Standard Institute (CLSI, unpublished data). For the purpose of harmonizing epidemiological reports on the basis of similar criteria so as to obtain comparable analyses of resistance patterns, the CLSI issued a specific guideline entitled Analysis and Presentation of Cumulative Antimicrobial Susceptibility Test Data, published in its fourth edition in January $2014(2,5)$.

This CLSI document is addressed to all professionals involved in test data processing and to software companies that wish to develop ad-hoc test data management software. It includes some specific recommendations for the collection, analysis and presentation of data and focuses in particular on the main critical issues, starting from strategies to eliminate multiple isolates of a microorganism from the same patient (multiple isolates). This article intends to review and expand the concepts covered in the CLSI guidelines, providing Italian clinical microbiologists with practical recommendations on how to manage the production, diffusion and assessment of epidemiological reports of isolated microorganisms and their antibiotic resistance patterns.

\section{Frequency of data analysis and reporting}

For the purpose of providing useful data to clinicians for the selection of empirical antibiotic therapies, reports should be issued at least annually.

Even more frequent analyses might be appropriate, provided the methodological requirements described in the next sections are met in 
the following instances: i) special clinical requirements (e.g. empirical therapy in intensive care units) or epidemiological requirements (e.g. within the framework of active surveillance programs); ii) identification of significant changes in the susceptibility data over the course of the year.

\section{Types of isolates to include in the analyses}

The calculation of susceptibility data should only consider samples collected for diagnostic purposes.

Any isolate from surveillance tests or screening tests to detect microorganisms with specific resistance phenotypes, such as methicillin-resistant Staphylococcus aureus (MRSA), vancomycin-resistant Enterococcus (VRE), carbapenemase-producing enterobacteria (CPE) should be excluded from analyses conducted on clinical isolates to avoid biases in the overall epidemiological data.

\section{Handling of data concerning duplicate isolates}

The most controversial issue regarding epidemiological data processing is the way in which multiple isolates of a given bacterial species from an individual patient (multiple isolates) are handled. In fact, they can be calculated with different algorithms that lead to different results: i) isolate-based approach: all isolates are equally considered; ii) patient-based approach: only the first isolate per patient is considered; iii) clinical episode-based approach: it considers all isolates from different episodes of infection (with a potential distinction by site of infection, resistance phenotype or period between two isolation dates); iv) resistance phenotype-based approach: it considers isolates with different phenotypic characteristics in terms of antibiotic susceptibility.

The impact of the different calculation strategies on the evaluation of percentage susceptibilities can be seen in an example of subsequent isolates of Staphylococcus aureus from a single patient (Table 1) and in the different methods of handling multiple isolates (Table 2).

Other data reported in the CLSI guidelines also prompt some general considerations on the effects of the selected calculation method: i) the isolate-based approach generates lower percentage susceptibilities, above all for hospital pathogens which can often cause prolonged infections and multiple positive cultures; ii) patient-, episode- and phenotype-based approaches generate more comparable data, however the episode- and phenotype-based approaches yield lower percentage susceptibilities, as they can better reflect isolates that develop resistances; iii) the difficulty of defining episodes and phenotypes can have a significant impact, above all for microorganisms responsible for hospitalacquired infections.
For the considerations presented above, the CLSI guidelines and the European Antimicrobial Resistance Surveillance Network (EARS-Net) guidelines recommend adopting the approach that considers the first isolate per patient, as it offers some practical advantages (4): i) it is an approach with direct relevance to guiding the selection of the empirical antibiotic treatment; ii) it is less complex to implement compared with phenotype- and episode-based approaches, which require definitions that are hard to generalize and can yield results which may be confusing and difficult to compare between different settings.

On the other hand, isolate-, episode- and phenotype-based approaches can be more useful in patients who developed infections during a hospital stay, above all long-term ones. However, it should be emphasized that no option can be actually deemed more or less appropriate and it should be recognized that any option selected leads to conceptually different data.

The Italian guidelines proposed by the Associazione Prevenzione Studio Infezioni (APSI), i.e. association for the prevention and the study of infections, recommend considering the first isolate within an interval of 30 days (3). For the purpose of improving the management of healthcare-associated infections from the clinical and epidemiological point of view, it might also be appropriate to consider the first antibiotype (susceptibility pattern of the strain) isolated at every site that can be correlated to a different infection over the course of 30 days. If this approach is applied to the above-mentioned example, all four isolates of the patient would be included in the epidemiological evaluation. In fact, the first is a methicillin-susceptible Staphylococcus aureus (MSSA) isolated from a wound infection, the second is a MRSA isolated from an episode of bacteremia, whereas the third and the fourth show two different antibiotypes (gentamicin-susceptible MRSA and gentamicin-resistant MRSA), also associated again with wound infection.

Whatever calculation strategy is adopted, it is recommended that this should be stated in the report, so that it can considered when comparing data generated from different strategies.

\section{Minimum number of isolates}

In order to have reliable susceptibility data, it is advisable to generate a cumulative antibiogram only for the species with at least 30 isolates tested in the relevant period. Other antibiotic resistance surveillance protocols, such as the EARS-Net protocol (4), already consider 20 isolates as a significant number.

The collection of a minimum number of isolates for a given species could be a concern in particular for smaller laboratories and infrequently isolated microorganisms. In these cases it may be appropriate to consider isolates collected over a longer period of time or to combine data from several facilities from the same geographic area or different microorganisms with similar features.

Table 1. Example of multiple isolates of Staphylococcus aureus from an individual patient with the corresponding susceptibility test results.

\begin{tabular}{|c|c|c|c|c|c|c|c|c|}
\hline \multirow[t]{2}{*}{ Isolate } & \multirow[t]{2}{*}{ Day } & \multirow[t]{2}{*}{ Site of collection } & \multicolumn{6}{|c|}{ Tested antibiotics } \\
\hline & & & Clin & Eri & Gen & Pen & Oxa & Van \\
\hline 1 & 1 & Wound & S & S & S & $\mathrm{R}$ & S & S \\
\hline 2 & 7 & Blood & $\mathrm{R}$ & $\mathrm{R}$ & $\mathrm{R}$ & $\mathrm{R}$ & $\mathrm{R}$ & S \\
\hline 3 & 20 & Wound & $\mathrm{R}$ & $\mathrm{R}$ & S & $\mathrm{R}$ & $\mathrm{R}$ & S \\
\hline 4 & 32 & Wound & $\mathrm{R}$ & $\mathrm{R}$ & $\mathrm{R}$ & $\mathrm{R}$ & $\mathrm{R}$ & S \\
\hline
\end{tabular}

Modified from Hindler et al. 2007 (5)

Clin, Clindamycin; Eri, Eritromycin; Gen, Gentamicin; Pen, Penicillin; Oxa, Oxacillin; Van, Vancomycin. 


\section{Antimicrobial agents to be analyzed}

The antibiotic resistance reports should present only antimicrobial agents that are routinely tested and clinically useful to clinicians. Therefore, they cannot include antimicrobial agents that may be effective in vitro against a given microorganism, but are not yet approved for therapeutic purposes.

In the case of second- or third-line antimicrobial agents, which are often subject to selective reporting or masking of the antibiogram, it is important to generate data which are not biased as a result of these practices. In fact, if susceptibility data of antimicrobial agents subject to routine selective reporting are evaluated considering only the strains with the highest resistance profiles, for which these antimicrobial agents are unmasked, susceptibility rates might be overestimated. For these molecules, therefore, data should be evaluated considering all tested isolates and not only those with a susceptibility rate in the antibiogram report. When this is not technically feasible, it can be acceptable to present the data generated from these kinds of tests, provided the adopted criteria are clearly indicated in the report. In some cases it might be correct to consider all isolates for which this molecule is masked as susceptible to the antimicrobial agent under examination. For instance, if the laboratory uses a selective reporting strategy that does not report carbapenems in the antibiogram for isolates of Escherichia coli susceptible to ampicillin, at the time of the evaluation all these isolates could be considered as susceptible to carbapenems.

\section{Interpretation and reporting of susceptibility tests}

According to the CLSI, susceptibility data should be reported in the cumulative antibiogram in terms of percentage of susceptible isolates $(\% \mathrm{~S})$, excluding the strains with intermediate susceptibility (except for some microorganisms listed below), since clinicians and pharmacists tend to focus on agents with the highest potential therapeutic success rate.

On the other hand, many international reports (EARS-Net, northern European countries, etc.) indicate rates of isolates that are resistant (R) or with intermediate susceptibility (I), taking into account that the definitions of multi-drug resistant (MDR) and extensively-drug resistant (XDR) are actually referred to resistance. For this reason, it is more common in Italy to report the percentages of non susceptible isolates ( $\% \mathrm{R}$ or $\% \mathrm{I} / \mathrm{R}$ ). In some cases, the distinction between I or $\mathrm{R}$ isolates can also be useful both from the epidemiological and clinical point of view.

Further evaluations must be done using the interpretation criteria (breakpoints and rules) adopted at the time of the analysis.

The comparison of data generated on the basis of different criteria (e.g. different breakpoints) would imply that quantitative data (minimum inhibitory concentration or inhibition zone diameters) are pre- served and reinterpreted with the updated criteria. When the epidemiology data software cannot reprocess data in this manner, use is recommended of footnotes that explain the methodological issue and allow correct evaluation of data, indicating that susceptibility data are not directly comparable, as they derive from the application of different breakpoints.

\section{Data validation}

Where epidemiology data software is used to generate the reports, CLSI guidelines suggest it should be validated, at least the first time it is used and after any change in the interpretation criteria, by comparing data generated manually.

The validation procedure requires that data for a given period are generated both manually and automatically so as to generate between 20 and 100 consecutive isolates for the species which often give multiple isolates (Staphylococcus aureus, Pseudomonas aeruginosa, Acinetobacter baumannii); the results should then be compared in terms of: i) number of patients; ii) susceptibility rate per each antimicrobial agent.

It is also possible to perform the validation by comparing the implemented software with a different software tool (e.g. software of the antibiogram tool).

\section{Indications concerning specific microorganisms (species or resistance mechanisms)}

The CLSI guidelines suggest using specific data presentation formats for some species.

Table 2. Isolates included in the analysis using the various algorithms to handle multiple isolates.

\begin{tabular}{lc} 
Algorithm & $\begin{array}{c}\text { Isolates included } \\
\text { in the analysis }\end{array}$ \\
\hline $\begin{array}{l}\text { Isolate-based (all isolates) } \\
\text { Patient-based (1 }{ }^{\text {st }} \text { isolate per patient) }\end{array}$ & $1,2,3,4$ \\
\hline Episode-based & 1 \\
- 7-day interval from 1 $1^{\text {st }}$ isolate & $1,3,4$ \\
- 7-day interval from previous isolate & $1,3,4$ \\
- 30-day interval from 1 $1^{\text {st }}$ isolate & 1,4 \\
- 30-day interval from 1 $1^{\text {st }}$ isolate & 1 \\
Resistance phenotype based (1 $1^{\text {st }}$ isolate per antibiotype) & $1,2,3$ \\
\hline Modified from Hindler et al. 2007 (5) &
\end{tabular}

Table 3. Example of susceptibility data for Klebsiella pneumoniae isolates stratified by resistance mechanism.

\begin{tabular}{|c|c|c|c|c|c|c|c|c|c|c|c|}
\hline \multirow[t]{2}{*}{ K. pneumoniae } & \multirow[t]{2}{*}{ Isolates } & \multicolumn{10}{|c|}{$\%$ susceptibility } \\
\hline & & Tzp & Ctx & Ctz & Cip & Gen & Ak & Ert & Mer & Tig & Col \\
\hline All isolates & 755 & 44 & 45 & 43 & 43 & 68 & 59 & 59 & 63 & 72 & 94 \\
\hline ESBL-producing isolates & 129 & 9 & 5 & 0 & 3 & 30 & 86 & 81 & 100 & 60 & 100 \\
\hline Carbapenemase-producing isolates & 306 & 0 & 0 & 0 & 0 & 48 & 6 & 0 & 8 & 32 & 86 \\
\hline ESBL- and carbapenemase- negative isolates & 320 & 92 & 100 & 100 & 93 & 98 & 99 & 100 & 100 & 100 & 100 \\
\hline
\end{tabular}

Tzp, Piperacillin-tazobactam; Ctx, Cefotaxime; Ctz, Ceftazidime; Cip, Ciprofloxacin; Gen, Gentamicin; Ak, Amikacin; Er, Ertapenem; Mer, Meropenem; Tig, Tigecycline; Col, Colistin. 
In particular: i) for Streptococcus pneumoniae: calculate the percentage of intermediate isolates susceptible to penicillin and the percentage of isolates susceptible to cefotaxime (or ceftriaxone), using both the breakpoints for isolates from the cerebrospinal fluid and isolates from other materials (with the EUCAST interpretative criteria only applicable to penicillin); ii) for viridans group streptococci: indicate both the percentage of intermediate strains and the percentage of strains susceptible to penicillin; iii) for Staphylococcus aureus: indicate the susceptibility rates of the different agents with respect to both total isolates and the subset of MSSA and MRSA.

In epidemiological settings where extended-spectrum beta-lactamase (ESBL) producing Enterobacteriaceae and/or CPE have become endemic, it could be useful to stratify data by resistance mechanism.

For instance, data of Klebsiella pneumoniae isolates could be expressed on the basis of 4 different categories (Table 3): i) total isolates; ii) ESBL-producing isolates; iii) carbapenemase-producing isolates; iv) ESBL- and carbapenemase-negative isolates.

In order to guide the empirical treatment of infection in which the etiological agent requires combination therapies, it could be useful to review the susceptibility rates to at least one of the antimicrobial agents considered in the combination. In this manner the susceptibility rate of the combination might help identify the most active drug combination against the target pathogen. For instance for Pseudomonas aeruginosa, it could be useful to examine the activity in a combination of ceftazidime+ciprofloxacin, imipenem+ciprofloxacin, ceftazidime+tobramycin, etc (Table 4).

Regarding microorganisms for which the treatment choice can depend also on the minimum inhibitory concentration (MIC) (e.g. vancomycin vs MRSA), it can be very useful to present the MIC distribution of that microorganism-antibiotic combination in its epidemiological setting to select an appropriate empirical therapy (Figure 1).

Although complex to understand for most clinicians, another potentially useful quantitative data approach, in particular for multidrugresistant (MDR) pathogens, is based on the calculation of the MIC90 (MIC value that inhibits the growth of $90 \%$ of isolates belonging to the strain under examination).

\section{Data stratification}

Numerous studies have demonstrated that the overall susceptibility rate can include subsets of extremely diversified data depending on multiple parameters $(1,6,7,8)$. Binkley et al. evaluated about 10,000 bacterial isolates and found that the resistance rates were significantly higher in patients in intensive care units than in patients in other units (1). Later, even though in a setting with a low resistance rate, Kuster et al. found very significant differences in the susceptibility to fluoroquinolones in Escherichia coli and to imipenem in Pseudomonas aeruginosa among strains isolated from patients in different units (6).

Reporting only the total rate might therefore be of little use as a support for informed decision-making in the selection of an empirical antimicrobial therapy.
To encourage the use of reports by clinicians, it can therefore be useful to stratify data on the basis of: i) patient profile (e.g.: age range); ii) healthcare setting (e.g.: outpatient units, nursing homes, hospital general wards, intensive care units, acute care units, long-term care units); iii) length of stay in hospital; iv) type of materials (e.g.: blood, urine, respiratory specimens).

Regarding the stratification of materials, the following homogenous groups of the main biological specimen are proposed: i) blood and vascular access devices: cultures of peripheral blood and central venous blood, vascular catheters; ii) lower respiratory tract specimen: tracheal aspirate, bronchial aspirate, brushing, bronchial washing, bronchoalveolar lavage, pleural fluid; iii) urine: urine, catheter urine; iv) pus and exudates: purulent materials, wound swabs, ulcers, ascitic fluid, peritoneal fluid, pericardial fluid; v) central nervous system (CNS): cerebrospinal fluid, ventricular drains.

The recent revision of the CLSI guidelines also suggests stratifying data on the basis of the various clinical needs.

\section{Data aggregation}

The presentation of susceptibility/resistance data by groups of microorganisms (e.g. Gram-negative bacteria, Enterobacteriaceae, Gram-positive cocci, staphylococci, etc.) can provide clinicians with a broader spectrum of information that can better reflect their actual clinical and etiological variability and therefore be more useful in empirical antimicrobial therapy decision-making (Table 5).

The CLSI guidelines suggest considering, for instance, the aggregation of susceptibility data of all Gram-negative bacteria isolated from blood.

In this kind of evaluation, besides the level of aggregation adopted, it is crucial to take into account the healthcare setting to which data are

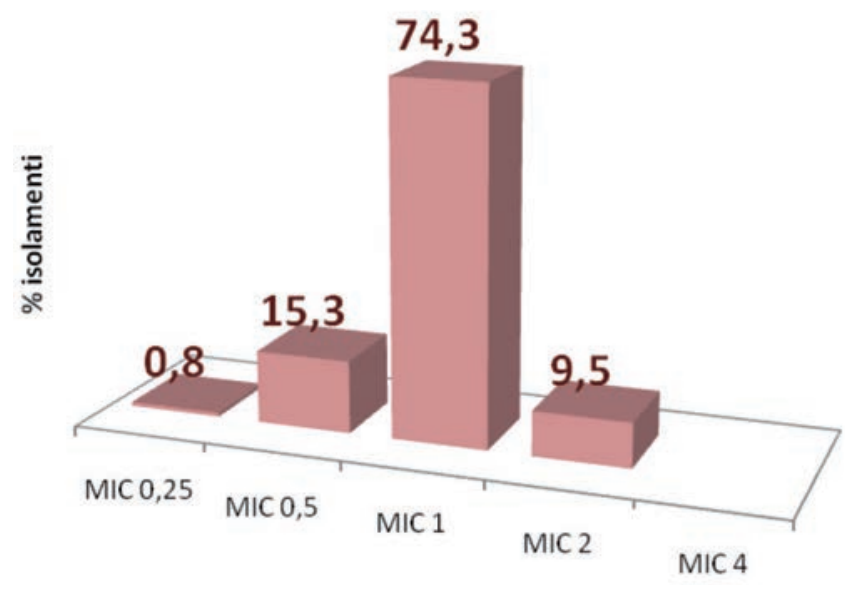

Figure 1. Example of a MIC distribution chart for vancomycin in methicillin-resistant Staphylococcus aureus isolates.

Table 4. Example of susceptibility data for Pseudomonas aeruginosa isolates to individual antibiotic agents and to some commonly used combinations in the anti-Pseudomonas therapy

\begin{tabular}{|c|c|c|c|c|c|c|c|c|c|c|c|}
\hline \multirow{2}{*}{\multicolumn{2}{|c|}{ Isolates }} & \multicolumn{10}{|c|}{$\%$ susceptibility } \\
\hline & & Trp & Ctz & Cip & Ak & Mer & Ctz+Cip & Mer+Cip & Mer+Ak & $\mathrm{Cip}+\mathrm{Ak}$ & Tzp+Ak \\
\hline Pseudomonas aeruginosa & 648 & 63 & 71 & 64 & 81 & 71 & 79 & 76 & 86 & 83 & 84 \\
\hline
\end{tabular}

Tzp, Piperacillina-tazobactam; Ctz, Ceftazidime; Cip, Ciprofloxacin; Ak, Amikacin; Mer, Meropenem. 
referred. The percentage distribution of the different microbial strains can differ significantly depending on whether it is associated with outpatients, inpatients, patients in intensive care units or in other hospital wards and, in the case of isolates from the urinary tract, whether patients are with or without catheters. If the scope of the analysis is not carefully identified, the resulting data might be misleading.

\section{Analyses of resistance patterns}

To evaluate the resistance pattern over time, use is recommended of ad-hoc tables and charts (e.g.: Figure 2) that compare data for the last period versus data of the previous periods. This approach can make it easier to identify the correlation with any change in the consumption of antimicrobial agents or to highlight any variation in the spread of particular multi-resistant microorganisms.

\section{Confidence intervals and statistical significance of changes in the susceptibility rates of isolates}

Given that the evaluation of antibiotic resistance is always very important from the microbiological point of view, its use in empirical antibiotic therapy decision-making will be all the more significant if data regarding isolated microorganisms are representative of the populations of bacteria that cause infections. It is therefore crucial to apply a statistical methodology to cumulative antibiograms. It will be especially useful for reports of this kind: i) to determine confidence intervals (IC) that quantify the accuracy of susceptibility percentages estimated on the basis of the sample size: for instance, if a laboratory tests 10 isolates of Enterobacter cloacae and 9 are susceptible to gentamicin, then the observed percentage susceptibility is $90 \%$. If the isolates tested are representative of a broader population of Enterobacter cloacae, the application of a $95 \%$ IC indicate that one can be $95 \%$ certain that the true percentage susceptibility lies between $55 \%$ and $100 \%$. However, if 1000 isolates were tested and 900 were found to be susceptible, while the observed percentage susceptibility would still be $90 \%$, the $95 \% \mathrm{CI}$ would between $88 \%$ and $92 \%$; ii) to evaluate the statistical significance of the different percentage susceptibilities observed in different periods: a consolidate approach uses the Chi-square test, considering $\mathrm{P} \leq 0.05$ as the significance level.

In this respect it is advisable to use tables such as those reported in the latest CLSI document, which facilitate the calculation of 95\% CIs (Table 6) and improve statistical significance (Table 7).

However, the notion of statistical significance should not be confused with the clinical and epidemiological value of data. In fact, if the number of isolates is high, even a small variation of the susceptibility rate might be statistically significant, yet without impacting on the choice of treatment by clinicians. Vice versa, if the number is low, higher percentages might not be statistically significant, but they should not be underestimated, since they may represent a new pattern of a given resistance.

\section{Data limits}

Antimicrobial susceptibility data are obviously calculated on the basis of the results of cultures tested by each individual laboratory and can therefore be affected by different sample collection and shipment policies.

The value of these data in empirical antibiotic therapy decisionmaking might be compromised where clinical samples are poorly representative of the typical patient for that type of infection. For

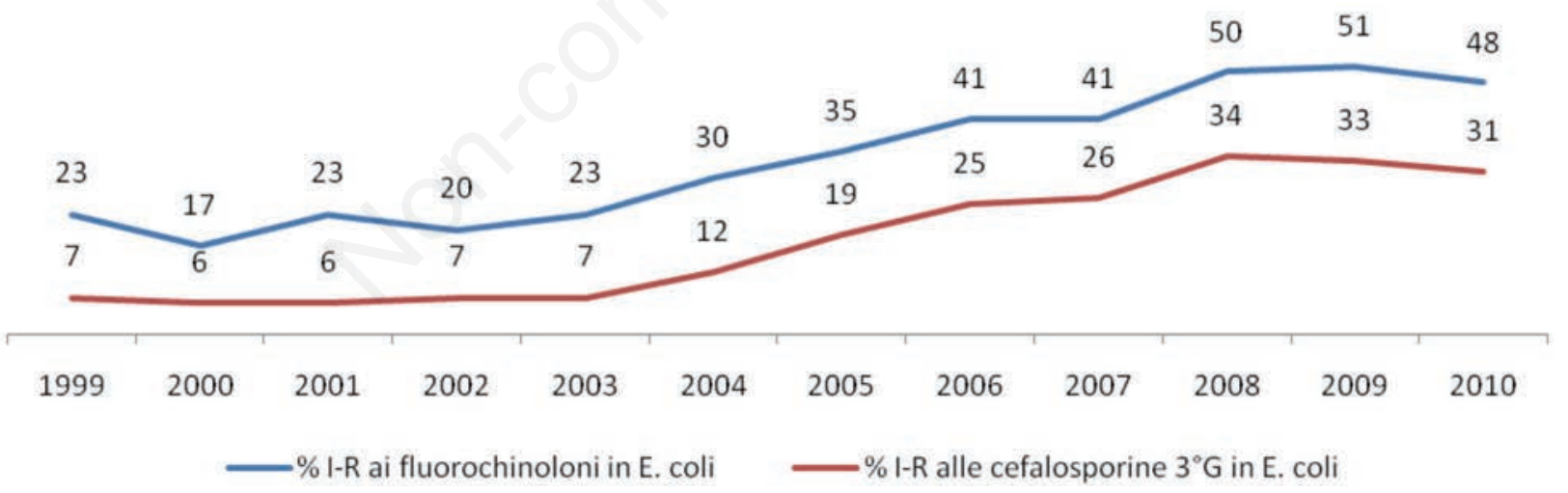

Figure 2. Example of a chart for monitoring the patterns of resistance phenotypes of particular clinical relevance over time.

Table 5. Examples of susceptibility data for isolates from Escherichia coli blood cultures and total gram-negative bacteria.

\begin{tabular}{|c|c|c|c|c|c|c|c|c|}
\hline & olates & & & & cept & & & \\
\hline & & Tzp & Ctx & Caz & Cip & Gen & Ak & Mer \\
\hline Escherichia coli & 76 & 81 & 79 & 79 & 74 & 89 & 100 & 100 \\
\hline All Gram-negative bacteria & 239 & 77 & 60 & 64 & 72 & 82 & 97 & 94 \\
\hline
\end{tabular}

Amp, Ampicillin; Amc, Amoxicillin-clavulanic acid; Tzp, Piperacillin-tazobactam; Ctx, Cefotaxime; Caz, Ceftazidime; Cip, Ciprofloxacin; SXT, Trimethoprim-sulfamethoxasole; Gen, Gentamicin; Ak, Amikacin; Mer, Meropenem. 
instance, the evaluation of the susceptibility rates might be biased by the fact that tests tend to be performed more often in patients with a previous antibiotic therapy failure or, more generally, in patients who spend long periods of time in hospital. Resistance data might therefore be higher compared with those obtained from all patients affected by the infection.

\section{Distribution of reports and data reviews}

The CLSI guidelines offer some indications for the distribution of reports, ideally in pocket guides or web-site materials. It also highly recommends distributing them via e-mail to potentially concerned healthcare providers (physicians, biologists, pharmacists, etc.), both to favor extensive and immediate access to these data and to avoid using paper materials.

Furthermore, it is suggested that data should be directly presented to the healthcare providers who are most involved in programs for an appropriate use of antibiotic therapy and for the control of healthcareassociated infections. It is also considered useful to present and discuss them with clinicians during ad-hoc meetings.

Finally, it seems reasonable to encourage the use of data presentation formats that can convey the message to clinicians in the simplest and more direct manner, for instance using ideal charts instead of classical tables.

Table 6. 95\% confidence intervals for various sample sizes (calculated using the Clopper-Pearson method): left columns include the lowest limits of confidence intervals, whereas right columns include the highest limits. For instance, in case of a $90 \%$ susceptibility for a sample including 50 isolates, the $95 \%$ confidence interval is between $78 \%$ and $97 \%$, i.e. a $90 \%$ susceptibility in a sample of 50 isolates has a $95 \%$ probability of being between $78 \%$ and $97 \%$.

\begin{tabular}{|c|c|c|c|c|c|c|c|c|c|c|c|c|c|c|c|c|c|c|}
\hline \multirow{3}{*}{$\begin{array}{l}\text { Sample sive } \\
10\end{array}$} & \multicolumn{18}{|c|}{ Susceptibility or resistance level } \\
\hline & \multicolumn{2}{|c|}{$10 \%$} & \multicolumn{2}{|c|}{$20 \%$} & \multicolumn{2}{|c|}{$30 \%$} & \multicolumn{2}{|c|}{$40 \%$} & \multicolumn{2}{|c|}{$50 \%$} & \multicolumn{2}{|c|}{$60 \%$} & \multicolumn{2}{|c|}{$70 \%$} & \multicolumn{2}{|c|}{$80 \%$} & \multicolumn{2}{|c|}{$90 \%$} \\
\hline & 0 & 45 & 3 & 56 & 7 & 65 & 12 & 74 & 19 & 81 & 26 & 88 & 35 & 92 & 44 & 97 & 55 & 100 \\
\hline 20 & 1 & 32 & 6 & 44 & 12 & 54 & 19 & 64 & 27 & 73 & 36 & 81 & 46 & 88 & 56 & 94 & 68 & 99 \\
\hline 30 & 2 & 27 & 8 & 39 & 15 & 49 & 23 & 59 & 31 & 69 & 41 & 77 & 51 & 85 & 61 & 92 & 73 & 98 \\
\hline 40 & 3 & 24 & 9 & 36 & 17 & 47 & 25 & 57 & 34 & 66 & 43 & 75 & 53 & 83 & 64 & 91 & 76 & 97 \\
\hline 50 & 3 & 22 & 10 & 34 & 18 & 45 & 26 & 55 & 36 & 64 & 45 & 74 & 55 & 82 & 66 & 90 & 78 & 97 \\
\hline 60 & 4 & 21 & 11 & 32 & 18 & 43 & 28 & 53 & 37 & 63 & 47 & 72 & 57 & 81 & 68 & 89 & 79 & 96 \\
\hline 70 & 4 & 20 & 11 & 31 & 20 & 42 & 28 & 52 & 38 & 62 & 48 & 72 & 58 & 80 & 69 & 89 & 80 & 96 \\
\hline 80 & 4 & 19 & 12 & 30 & 20 & 41 & 29 & 52 & 39 & 61 & 48 & 71 & 59 & 80 & 70 & 88 & 81 & 96 \\
\hline 90 & 5 & 18 & 12 & 30 & 21 & 41 & 30 & 51 & 39 & 61 & 49 & 70 & 59 & 79 & 70 & 88 & 82 & 95 \\
\hline 100 & 5 & 18 & 13 & 29 & 21 & 40 & 30 & 50 & 40 & 60 & 50 & 70 & 60 & 79 & 71 & 87 & 82 & 95 \\
\hline 200 & 6 & 15 & 15 & 26 & 24 & 37 & 33 & 47 & 43 & 57 & 53 & 67 & 63 & 76 & 74 & 85 & 85 & 94 \\
\hline 400 & 7 & 13 & 16 & 24 & 26 & 35 & 35 & 45 & 45 & 55 & 55 & 65 & 65 & 74 & 76 & 84 & 87 & 93 \\
\hline 600 & 8 & 13 & 17 & 23 & 26 & 34 & 36 & 44 & 46 & 54 & 56 & 64 & 66 & 74 & 77 & 83 & 87 & 92 \\
\hline 1000 & 8 & 12 & 18 & 23 & 27 & 33 & 37 & 43 & 47 & 53 & 57 & 63 & 67 & 73 & 77 & 82 & 88 & 92 \\
\hline
\end{tabular}

Modified from Clinical and Laboratory Standards Institute, 2014 (2).

Table 7. Statistically significant changes in susceptibility rates of samples with a comparable size (if the susceptibility rate decreases, if the rate increases). Example: for a sample size of 100 isolates observed in two different periods with an $\mathbf{8 0} \%$ susceptibility in the first period, a decline of susceptibility to $\leq 66 \%$ or its increase to $\geq 91 \%$ will be considered statistically significant (ns: not significant).

\begin{tabular}{lccccccccccccccccc} 
\% initial & \multicolumn{1}{c}{ Susceptibility decrease, sample sive } & \multicolumn{4}{c}{ Susceptibility increase, sample sive } \\
susceptibility & 10 & 20 & 50 & 100 & 200 & 400 & 600 & 1000 & 10 & 20 & 50 & 100 & 200 & 400 & 600 & 1000 \\
98 & - & - & 84 & 90 & 93 & 95 & 95 & 96 & - & - & ns & ns & ns & 100 & 100 & 100 \\
95 & - & 65 & 78 & 85 & 89 & 91 & 92 & 92 & - & ns & ns & ns & 99 & 98 & 98 & 97 \\
\hline 90 & 30 & 55 & 72 & 78 & 82 & 85 & 86 & 87 & ns & ns & ns & 98 & 96 & 95 & 94 & 93 \\
80 & 20 & 45 & 60 & 66 & 71 & 73 & 75 & 76 & ns & ns & 96 & 91 & 88 & 86 & 85 & 84 \\
\hline 70 & 10 & 30 & 48 & 55 & 60 & 63 & 64 & 65 & ns & 100 & 88 & 83 & 80 & 77 & 76 & 75 \\
60 & 0 & 20 & 38 & 45 & 49 & 52 & 54 & 55 & ns & 95 & 80 & 75 & 70 & 67 & 66 & 65 \\
\hline 50 & 0 & 15 & 28 & 35 & 39 & 42 & 44 & 45 & 100 & 85 & 72 & 65 & 61 & 58 & 56 & 55 \\
40 & $\mathrm{~ns}$ & 5 & 20 & 25 & 30 & 33 & 34 & 35 & 100 & 80 & 62 & 55 & 51 & 48 & 46 & 45 \\
\hline 30 & $\mathrm{~ns}$ & 0 & 12 & 17 & 20 & 23 & 24 & 25 & 90 & 70 & 52 & 45 & 40 & 37 & 36 & 35 \\
20 & $\mathrm{~ns}$ & $\mathrm{~ns}$ & 4 & 9 & 12 & 14 & 15 & 16 & 80 & 55 & 40 & 34 & 29 & 27 & 25 & 24 \\
\hline 10 & $\mathrm{~ns}$ & $\mathrm{~ns}$ & $\mathrm{~ns}$ & 2 & 4 & 5 & 6 & 7 & 70 & 45 & 28 & 22 & 18 & 15 & 14 & 13 \\
\hline
\end{tabular}

Modified from Clinical and Laboratory Standards Institute, 2014 (2). 


\section{Comitato CoSIAS-AMCLI}

Committee for the study of healthcare-associated infections of the Associazione Microbiologi Clinici Italiani (AMCLI), i.e. Association of Italian Clinical Microbiologists: Mario Sarti, Simone Ambretti, Giancarlo Basaglia, Susanna Cuccurullo, Carla Fontana, Lucina Fossati, Barbara Pieretti, Carlo Tascini.

\section{References}

1. Binkley S, Fishman NO, LaRosa LA, et al. Comparison of unit-specific and hospital-wide antibiograms: potential implications for selection of empirical antimicrobial therapy. Infect Control Hosp Epidemiol 2006; 27: 682-7.

2. Clinical Laboratory Standard Institute. Analysis and presentation of cumulative antimicrobial susceptibility test data; approved guideline. 4th edition. 2014 CLSI document M39-A4. Wayne, PA: Clinical and Laboratory Standards Institute.

3. De Paoli P, Goglio A, Nicoletti P. Proposta di linee guida per l'analisi e la presentazione dei risultati cumulativi degli antibiogrammi. Associazione per la Prevenzione ed il Controllo delle Infezioni
(APSI). Giornale Italiano delle Infezioni Ospedaliere 2002; 9: 66-73.

4. European Centre for Disease Prevention and Control. Antimicrobial resistance surveillance in Europe 2012. 2013 Annual Report of the European Antimicrobial Resistance Surveillance Network (EARSNet). Available from: http://ecdc.europa.eu/en/publications/ Publications/antimicrobial-resistance-surveillance-europe-2013.pdf

5. Hindler JF, Stelling J. Analysis and presentation of cumulative antibiograms: a new consensus guideline from the Clinical and Laboratory Standards Institute. Clin Infect Dis 2007; 44: 867-73.

6. Kuster SP, Ruef C, Zbinden R, et al. Stratification of cumulative antibiograms in hospitals for hospital unit, specimen type, isolate sequence and duration of hospital stay. J Antimicrob Chemother 2008; 62: 1451-61.

7. McGregor JC, Bearden DT, Townes JM, et al. Comparison of antibiograms developed for inpatients and primary care outpatients. Diagn Microbiol Infect Dis 2013; 76: 73-9.

8. Swami S, Banerjee R. Comparison of hospital-wide and age and location - stratified antibiograms of S. aureus, E. coli, and S. pneumoniae: age- and location-stratified antibiograms. Springer Plus $2013 ; 2: 63$.

9. World Health Organization. Prevention of hospital-acquired infections. A practical guide. $2^{\text {nd }}$ edition, 2002. Available from: http:// www.who.int/csr/resources/publications/whocdscsreph200212.pdf. 